\title{
Dynamical real space renormalization group applied to sandpile models
}

\author{
Eugene V. Ivashkevich, ${ }^{1}$ Alexander M. Povolotsky, ${ }_{1}^{1}$ Alessandro Vespignani, ${ }^{2}$ and Stefano Zapperi ${ }^{3}$ \\ ${ }^{1}$ Bogoliubov Laboratory of Theoretical Physics, JINR, Dubna 141980, Russia \\ ${ }^{2}$ The Abdus Salam International Centre for Theoretical Physics (ICTP), P.O. Box 586, 34100 Trieste, Italy \\ ${ }^{3}$ PMMH-ESPCI, 10 Rue Vauquelin, 75234 Paris Cedex 05, France
}

(Received 12 December 1997)

\begin{abstract}
A general framework for the renormalization group analysis of self-organized critical sandpile models is formulated. The usual real space renormalization scheme for lattice models when applied to nonequilibrium dynamical models must be supplemented by feedback relations coming from the stationarity conditions. On the basis of these ideas the dynamically driven renormalization group is applied to describe the boundary and bulk critical behavior of sandpile models. A detailed description of the branching nature of sandpile avalanches is given in terms of the generating functions of the underlying branching process. [S1063-651X(99)06006-7]

PACS number(s): 05.65.+b, 05.40.-a, 05.70.Ln
\end{abstract}

\section{INTRODUCTION}

In the past decade it has been recognized that a fair amount of physical phenomena are characterized by strong fluctuations and long-range correlation functions. According to the theory of equilibrium statistical physics, we expect scale invariance only in the presence of certain symmetries or at critical points [1]. We are therefore led to seek the origin of the scale invariance in nature, in the rich domain of nonequilibrium systems [2-5]. One might hope, in fact, that there might be classes of nonequilibrium systems that generate scale invariance for a wide (and arbitrary) range of physical parameters, providing an explanation for the commonly observed scaling laws.

Pursuing this aim, Bak, Tang, and Wiesenfeld proposed the concept of self-organized criticality (SOC) [6,7] as a unifying framework to describe a vast class of dynamically driven systems that evolve in a stationary state with a broad power law distribution of energy dissipating events. To illustrate the basic ideas of SOC, they introduced a cellular automaton model of sandpiles. In this model, criticality emerges if the system is driven at an infinitesimal rate [7-10]. Because of the enormous conceptual potentiality, SOC ideas have reverberated rapidly throughout the sciences, from geophysics to economics and biology, as a prototype mechanism to understand the manifestation of scale invariance and complexity in natural phenomena.

The major source of difficulties in the study of sandpile models is the absence of a general criterion, like the use of the Gibbs distribution in equilibrium systems, to assign an ensemble statistical measure to a particular configuration of the system. This problem is common to many nonequilibrium systems whose theoretical understanding lags far behind the equilibrium theory. In particular, many relations among sandpile automata and systems with a nonequilibrium absorbing critical point have been recently brought to light [10].

In the past years, we have developed a renormalization group (RG) strategy for sandpile models [11] that has also been applied [12] to forest-fire models [8,9]. This approach deals with the critical properties of the system by introducing in the renormalization equations a dynamical steady-state condition that provides the nonequilibrium stationary statistical weights to be used in the calculation. This scheme, named the dynamically driven renormalization group (DDRG) [13], has been successively generalized as a renormalization framework for systems with a nonequilibrium critical steady state. Recently, the DDRG approach has been improved including higher order proliferations through a general scheme [14]. The method has also been applied to one-dimensional sandpiles [15], directed sandpiles [16,17], and other nonequilibrium systems [18].

Here we discuss the application of the DDRG to sandpile models, deriving systematically the previous RG schemes $[11,14]$ and presenting extended results. We will introduce the general strategy of the DDRG for the critical height sandpile models, and its practical implementation for increasingly complex proliferation schemes. In order to treat such a high level of calculation complexity, we introduce a generating function for the basic recursion relations. The scheme is then extended by exploiting the analogy with a particular chain chemical reaction. Finally its application to the calculation of the boundary critical behavior is shown.

The paper is organized as follows. In Sec. II we introduce the class of sandpile automata and its mapping into a general nonequilibrium cellular automaton (CA). Section III presents the dynamically driven renormalization group general scheme. Section IV shows the explicit application of the DDRG to the sandpile in its simple scheme. In Sec. V we present the actual calculations of the renormalization equations and their generating function and results obtained. Section VI describes the extended chemical reaction scheme and its results. Section VII is devoted to the renormalization analysis of the boundary critical behavior. Section VIII presents the summary and conclusions.

\section{SANDPILE MODEL}

The prototype example for SOC is provided by sandpiles: sand is added grain by grain until unstable sand (a too large local slope of the pile) slides off. In this way the pile reaches a steady state, in which additional sand grains fall off the pile by avalanche events. The steady state is critical since avalanches of any size are observed. This class of models can be 
used to describe a generic avalanche phenomenon, interpreting the sand as energy, mechanical stress, or heat memory.

Sandpile models are cellular automata $[7,19]$ defined in a $d$-dimensional lattice. A discrete or continuous variable $E(i)$, which we denote by energy, is associated with each lattice site $i$. At each time step an input energy $\delta E$ is added to a randomly chosen site. When the energy on a site reaches a threshold value $E_{c}$, the site relaxes, transferring energy to the neighboring sites

$$
\begin{gathered}
E(i) \rightarrow E(i)-\sum_{e} \Delta E(e), \\
E(i+e) \rightarrow E(i+e)+\Delta E(e),
\end{gathered}
$$

where $e$ represents the unit vectors on the lattice. A typical choice for the parameters is, for example, $E_{c}=4$ and $\Delta E(e)=\delta E=1$, but other possibilities have also been considered. The relaxation of the first site can induce a series of relaxations generating an avalanche. Note that the energy is added to the system only when the configuration is stable (i.e., all the sites are below the threshold). The boundary conditions are usually chosen to be open so that energy can leave the system. Under these conditions the system organizes itself into a stationary state characterized by avalanche of all length scales. In particular, the distribution for avalanche sizes $s$ decays as a power law $P(s) \sim s^{-\tau}$, and the linear size of the avalanche scales with time $r \sim t^{z}$. This model has been extensively studied in the past by means of numerical simulations [20-23], and several exact results have been derived for Abelian sandpiles models (ASMs) [24-30].

Given the above definition of sandpiles we can rephrase them in the language of a discrete nonequilibrium probabilistic CA. To each site $i$ is associated a variable $s_{i}$ that can assume $q$ different values $\left(s_{i}=1,2,3, \ldots, q\right)$. For instance, each state might correspond to an allowed energy level. The subscript $i$ labels the lattice site. A complete set $s \equiv\left\{s_{i}\right\}$ of lattice variables specifies a configuration of the system. We define $\left\langle s|T(\mu)| s^{0}\right\rangle$ as the transition rate from a configuration $s_{0}$ to a configuration $s$ in a time step $t$ as a function of a set of parameters $\mu=\left\{\mu_{i}\right\}$. SOC automata are usually defined by a transition probability given by the product

$$
\left\langle s|T| s^{0}\right\rangle=\prod_{i=1}^{N} \tau\left(s_{i} \mid s_{i}^{0},\left\{s_{i+e}^{0}\right\}\right),
$$

where $N=L^{d}$ is the number of sites, and $e$ specifies the nearest neighbor (NN) vector. The dynamics is therefore expressed as a product of one-site transition probabilities, depending upon the site and its nearest-neighbor states at the previous time step.

As we said, the common characteristic of SOC systems is the presence of a nonequilibrium critical steady state, which we can analyze using the DDRG formalism. However, it is worth remarking that SOC systems reach true criticality just in the limit of an infinite slow driving condition. This means that the perturbing time scale is much larger than the dynamical activity one. SOC systems relax far more rapidly than they are perturbed. In practice, this implies that no new grain of sand is dropped until the avalanche started by the previous grain has finished. In this way avalanches cannot overlap, and their dynamics is well defined with respect to the external field. A complete RG analysis should also take into account the driving field. However, since we are interested in the critical point, we will study the system in the limit of slow driving. A more detailed discussion of the complete sandpile automaton phase diagram is provided in Ref. [10].

\section{DDRG}

The probability distribution of CAs such as those shown in the previous section obeys the following master equation (ME):

$$
P\left(s, t_{0}+t\right)=\sum_{\left\{s^{0}\right\}}\left\langle s|T(\mu)| s^{0}\right\rangle P\left(s^{0}, t_{0}\right) .
$$

The explicit solution of the master equation is not in general available, but we can extract the critical properties of the model by a renormalization group analysis. We coarse grain the system by rescaling lengths and time according to the transformation $x \rightarrow b^{-1} x$ and $t \rightarrow b^{-z} t$. The renormalization transformation is constructed through the operator $\mathcal{R}(S, s)$ that introduces a set of coarse grained variables $S \equiv\left\{S_{i}\right\}$ and rescales the lengths of the system [31]. In general, $\mathcal{R}$ is a projection operator with the properties $\mathcal{R}(S, s) \geqslant 0$ for any $\left\{S_{i}\right\},\left\{s_{i}\right\}$, and $\Sigma_{\{S\}} \mathcal{R}(S, s)=1$. These properties preserve the normalization condition of the renormalized distribution. The explicit form of the operator $\mathcal{R}$ is defined case by case in various applications of the method. Usually, it corresponds to a block transformation in which lattice sites are grouped together in a supersite that defines the renormalized variables $S_{i}$ by means of a majority or a spanning rule.

We subdivide the time step in intervals of the unitary time scale $\left(t_{0}=0\right)$, obtaining the coarse graining of the system as follows:

$$
P^{\prime}\left(S, t^{\prime}\right)=\sum_{\{s\}} \mathcal{R}(S, s) \sum_{\left\{s^{0}\right\}}\left\langle s\left|T^{b^{z}}(\mu)\right| s^{0}\right\rangle P\left(s^{0}, 0\right),
$$

where we have included the application of the operator $\mathcal{R}$ and $t^{\prime}=b^{z} t$. The meaning of $\left\langle s\left|T^{b^{z}}(\mu)\right| s^{0}\right\rangle$ has to be defined explicitly: the simplest possibility is $b^{z}=N$, where $N$ is an integer number and $T^{N}$ denotes the application of the dynamical operator $N$ times. In general, since we are dealing with a discrete time evolution, we have to consider $T^{b^{z}}$ as a convolution over different paths, chosen by an appropriate condition. The detailed definition of the effective operator $T^{b^{z}}$ for the sandpile is reported in the next section. By multiplying and dividing each term of Eq. (5) by $P^{\prime}\left(S^{0}, 0\right)$ $=\Sigma_{\left\{s^{0}\right\}} \mathcal{R}\left(S^{0}, s^{0}\right) P\left(s^{0}, 0\right)$ and using the properties of the operator $\mathcal{R}$, after some algebra we get 


$$
P^{\prime}\left(S, t^{\prime}\right)=\sum_{\left\{S^{0}\right\}}\left(\frac{\sum_{\left\{s^{0}\right\}} \sum_{\{s\}} \mathcal{R}\left(S^{0}, s^{0}\right) \mathcal{R}(S, s)\left\langle s\left|T^{b^{z}}(\mu)\right| s^{0}\right\rangle P\left(s^{0}, 0\right)}{\sum_{\left\{s^{0}\right\}} \mathcal{R}\left(S^{0}, s^{0}\right) P\left(s^{0}, 0\right)}\right) P^{\prime}\left(S^{0}, 0\right),
$$

which finally identifies the renormalized dynamical operator $\left\langle S\left|T^{\prime}\right| S^{0}\right\rangle$. In other words, the new dynamical operator $T^{\prime}$ is the sum over all the dynamical paths of $b^{z}$ steps that from a starting configuration $\left\{s_{i}^{0}\right\}$ lead to a configuration $\left\{s_{i}\right\}$ that renormalizes in $\left\{S_{i}^{0}\right\}$ and $\left\{S_{i}\right\}$, respectively. The sum is weighted by the normalized statistical distribution of each configuration. The scheme discussed so far is a general formulation, and its application to systems that exhibit a stationary state presupposes knowledge of the explicit form of the steady-state distribution $W(s)=P(s, t \rightarrow \infty)$. For instance, in equilibrium phenomena $W(s)$ is given by the Gibbs distribution. In this case it is possible to apply several methods such as cumulant expansions and exact or approximate decimation to obtain the form of the recursion relations. For nonequilibrium dynamical systems, in general, we do not know the form of the steady-state distribution. We will therefore develop an approximate method to evaluate the stationary distribution to be used in the calculation of the renormalized master equation.

The steady-state distribution can in general be split into two parts,

$$
W(s)=W^{(i)}(s)+W^{(c)}(s)
$$

where $W^{(i)}(s)$ and $W^{(c)}(s)$ are, respectively, the incoherent and coherent part of the distribution. The incoherent part of the distribution does not include correlations among variables and expresses a mean field approximation for the system. The coherent part $W^{(c)}(s)$ can be subdivided into parts describing different kinds of correlations: nearest neighbors, next-nearest neighbors, etc. The incoherent part is a factorized distribution that, for systems characterized by a $q$-state variable (see Sec. II), has the form

$$
W^{(i)}(s)=\prod_{i}\left\langle\rho_{s_{i}}\right\rangle,
$$

where $\left\langle\rho_{\kappa}\right\rangle$ is the average density of sites in the $\kappa$ state. In this way, we have approximated the probability of each configuration $\left\{s_{i}\right\}$ as a product measure of the mean field probability having a state $s_{i}$ in each corresponding site. The incoherent part contribution to the renormalization equation can be obtained by stationarity conditions for the system $\mathcal{S}_{\mu}\left(\left\{\left\langle\rho_{\kappa}\right\rangle\right\}\right)=0$ to evaluate the densities $\left\langle\rho_{\kappa}\right\rangle$. These conditions are derived from dynamical mean field equations that describe the driving of the system to the nonequilibrium steady state by means of balance constraints. The operator $\mathcal{S}_{\mu}$ depends upon the same dynamical parameters of the operator $T$, and by solving the stationary condition equation, the average densities of the $\kappa$ states for the coarse grained system are obtained as a function of $\mu$ at the corresponding iteration of the RG equations. By inserting the approximate distribution in Eq. (6), we thus get the following set of renormalization equations:

$$
\begin{gathered}
\left\langle S\left|T^{\prime}(\mu)\right| S^{0}\right\rangle \\
=\frac{\sum_{\left\{s^{0}\right\}} \sum_{\{s\}} \mathcal{R}\left(S^{0}, s^{0}\right) \mathcal{R}(S, s)\left\langle s\left|T^{b^{z}}(\mu)\right| s^{0}\right\rangle \prod_{i}\left\langle\rho_{s_{i}^{0}}\right\rangle}{\sum_{\left\{s^{0}\right\}} \mathcal{R}\left(S^{0}, s^{0}\right) \prod_{i}\left\langle\rho_{s_{i}^{0}}\right\rangle}, \\
\mathcal{S}_{\mu}\left(\left\{\left\langle\rho_{\kappa}\right\rangle\right\}\right)=0,
\end{gathered}
$$

where the second equation denotes the dynamical steadystate condition that allows evaluation of the approximate stationary distribution at each coarse graining scale. We call Eq. (10) the driving condition, since it drives the RG equations acting as a feedback on the scale transformation. Equations (9) and (10) are the basic renormalization equations from which the desired recursion relations are derived. Imposing the requirement that the renormalized operator $T^{\prime}$ have the same functional form of the operator $T$, i.e., $T^{\prime}(\mu)=T\left(\mu^{\prime}\right)$, we obtain the rescaled parameter set $\mu^{\prime}=f(\mu)$. This implies that the renormalized single time distribution $P^{\prime}\left(S, t^{\prime}\right)$ has the same functional form of the original distribution $P(s, t)$. The critical behavior of the model is obtained by studying the fixed points $\mu^{*}=f\left(\mu^{*}\right)$. Since we are dealing with discrete evolution operators $T$, we define the time scaling factor $b^{z}$ as the average number of steps and apply the operator $T$ in order to obtain that $T^{\prime}(\mu)=T\left(\mu^{\prime}\right)$ for the coarse grained system. In this way we obtain a time recursion relation $t^{\prime}$ $=g(\mu) t$, or equivalently $b^{z}=g(\mu)$, from which it is possible to calculate the dynamical critical exponent $z=\ln g\left(\mu^{*}\right) / \ln b$. In this form of the DDRG, we take into account only the uncorrelated part of the steady-state probability distribution. The results obtained are not trivial because correlations in the systems are considered in the dynamical renormalization of the operator $T$, which given a starting configuration traces all the possible paths leading to the renormalized final configuration. Moreover, geometrical correlations are treated by the operator $\mathcal{R}$ that maps the system by means of spanning conditions or majority rules. The renormalized uncorrelated part of the stationary distribution is evaluated from the stationary condition with renormalized parameters, thus providing an effective treatment of correlations. One can then improve the results by including higher order contributions in the unknown stationary distribution $W(s)$ using cluster variation methods [32]. 


\section{RENORMALIZATION SCHEME FOR SANDPILE MODELS}

Here, we show in detail the DDRG scheme for sandpile models. For the sake of clarity, we start by considering the minimum proliferation scheme. A more refined scheme is discussed in the following sections.

To simplify the description of sandpile models as much as possible, we can reduce the number of states of each site in the following way. At any scale, we divide the sites into critical $\left(s_{i}=1\right)$ and stable $\left(s_{i}=0\right)$. Stable sites do not relax when energy is added to them. On the other hand, critical sites relax when they receive an energy grain $\delta E_{i n}$. In this formalism we define $\langle\rho\rangle$ as the density of critical sites. For convenience, we also define unstable sites $\left(s_{i}=2\right)$ as those that are relaxing, even though they are not present in the static configurations of the system. These definitions can be extended to a generic scale $b$. For instance, a cell at scale $b$ is considered critical if the addition of energy $\delta E_{i n}(b)$ induces a relaxation of the size of the cell (i.e., the avalanche spans the cell).

In a relaxation event at the minimal scale, energy is equally distributed in the four directions. This is no longer the case at a coarse grained level where different possibilities arise: the energy in principle can be distributed to one, two, three, or four neighbors. It is also worth remarking that in a certain case unstable sites at the coarse grained scale do not dissipate energy to nearest neighbors, representing just intrasite energy rearrangements. These processes define the probability that relaxation events will take place on the renormalized scale without energy transfer. All these events occur with probabilities

$$
\vec{P}=\left(p_{0}, p_{1}, p_{2}, p_{3}, p_{4}\right) .
$$

In terms of the matrix element $\langle 0|T| 2\rangle$ the vector $\vec{P}$ represents the probabilities

$$
p_{n}=\langle 0|T| 2\rangle_{n}
$$

where $\langle 0|T| 2\rangle_{n}$ is the probability that a relaxing site will become stable and transfer energy to $n$ neighbors. In this way, we have obtained the set of parameters that describes the dynamics. Of course, the choice of parameter space is not uniquely determined; one encounters proliferation problems typical of real space RG methods. For instance, higher order proliferations are due to multiple relaxations of the same site and sites becoming critical during the dynamical process (i.e., $\langle 1|T| 2\rangle$ ). In the following, the practical implementation of the method considers just the minimal proliferation we have reported above. In the next sections, a more refined scheme will be treated.

First of all, let us show how the driving condition is obtained by imposing the stationarity of the process. The average energy of a site evolves according to the following equation written in the continuum notation:

$$
\frac{d E(t)}{d t}=\delta E_{\text {in }}-\delta E_{\text {out }},
$$

where $\delta E_{\text {in }}$ is the average energy entering into the site either because of relaxation in a neighboring site or because of the external perturbations, and $\delta E_{\text {out }}$ is the average energy dissipated by the site. The stationary state is characterized by the balance between the energy that goes in and the energy that goes out of the system. We assume that energy is transferred in "quanta" $\delta E=\delta E_{i n}$ in each direction, and we on average obtain

$$
\delta E=\langle\rho\rangle \delta E \sum_{n} n p_{n}
$$

which implies

$$
\langle\rho\rangle=\frac{1}{\sum_{n} n p_{n}} .
$$

This relation gives the average density of critical sites in the steady state, allowing us to evaluate the approximate stationary distribution at each scale. It is worth remarking that the above relation is not able to take into account how the stationary state is approached and the role of boundary conditions or driving on it. Nevertheless, the stationary state bulk properties are expected to be well approximated without considering these details.

The renormalized matrix element is then obtained by considering all the renormalized processes that span the cell and transfer energy outside,

$$
p_{n}^{\prime}=\left\langle S_{i}=0\left|T^{\prime}\right| S_{i}^{0}=2\right\rangle_{n} .
$$

We proceed in defining explicitly a renormalization procedure for the dynamics by considering a finite truncation on four-site cells. This corresponds to a cell-to-site transformation on a square lattice, in which each cell at the coarser scale is formed by four subcells at the finer scale: the length scaling factor is $b=2$. In this case, the operator $\mathcal{R}$ can be written in the following way:

$$
\mathcal{R}(S, s)=\prod_{J} \mathcal{R}\left(S_{J},\left\{s_{i}\right\}_{J}\right)
$$

where each term is acting on a specific cell $J$ and $\left\{s_{i}\right\}_{J}$ denotes the configurations of sites belonging to that cell. A cell is renormalized as a relaxing one if it contains a relaxing subcell that transfers energy to a critical subcell. In this way, we ensure that the occurring relaxation process extends over the size of the renormalized length scale independently of the successive avalanche evolution. A critical cell is therefore defined by a cell that can be spanned by a path of relaxation events. The scheme considers only connected paths that span the cell from left to right or top to bottom. This spanning rule implies that only paths extending over the size of the resulting length scale contribute to the renormalized dynamics, and it ensures the connectivity properties of the avalanche in the renormalization procedure.

Every cell at the coarser scale can be characterized by an index $\alpha$ that indicates the configuration of subcells, and we have that $\Sigma_{\left\{s_{i}\right\}} \rightarrow \Sigma_{\alpha}$. The approximated stationary distribution [Eq. (8)] for each of these configurations is given by 


$$
W_{\alpha}(\langle\rho\rangle)=n_{\alpha} \prod_{i=1}^{4}\left\langle\rho_{s_{i}}\right\rangle
$$

where $n_{\alpha}$ is a factor due to the multiplicity of each configuration.

By using this scheme and replacing sums over configurations with sums over the index $\alpha$, the recursion relations can then be rewritten in the simpler form

$$
p_{n}^{\prime}=\frac{1}{\mathcal{N}} \sum_{\alpha} W_{\alpha}(\langle\rho\rangle) \sum_{\alpha^{\prime}}\left\langle\alpha^{\prime}\left|T^{b^{z}}\left(p_{n^{\prime}}\right)\right| \alpha\right\rangle_{n},
$$

where $|\alpha\rangle,\left|\alpha^{\prime}\right\rangle$ denotes the four-site configurations that renormalize in $\left|S_{i}^{0}=2\right\rangle$ and $\left|S_{i}=0\right\rangle$, respectively. In the above expression the denominator of Eq. (9) is adsorbed in the normalization factor $\mathcal{N}$.

The effective operator $T^{b^{z}}$ contains all the dynamical processes that contribute to the definition of a meaningful renormalized dynamics. We define the following transformation:

$$
\left\langle s\left|T^{b^{z}}(\mu)\right| s^{0}\right\rangle=\sum_{N} \mathcal{D}_{N}\left\langle s\left|T^{N}(\mu)\right| s^{0}\right\rangle,
$$

where $\mathcal{D}_{N}$ is the renormalization operator for the dynamical evolution of the system: it is a projection operator that samples only the paths of $N$ time steps that have to be considered in the definition of the effective operator $T^{b^{z}}$.

The operator $\mathcal{D}_{N}$ is chosen on the basis of physical considerations: spanning conditions, etc. In addition, $\mathcal{D}_{N}$ should satisfy some general properties in order to preserve the symmetry or the internal space of the dynamical variables. For instance, we have to ensure the normalization of the effective dynamical operator by the property

$$
\sum_{\{s\}} \sum_{N} \mathcal{D}_{N}\left\langle s\left|T^{N}(\mu)\right| s^{0}\right\rangle=1
$$

Moreover, $\mathcal{D}_{N}$ must be consistent with the definition of the renormalization operator $\mathcal{R}$ : it should describe dynamical processes among renormalized variables of the same type as those given by the operator $\mathcal{R}$. Finally, $\mathcal{D}_{N}$ has to preserve the form of the dynamical operator $T$ at each scale. This condition requires that the time scaling be consistent with the length scaling used in $\mathcal{R}$. In this way, it is possible to map the renormalized system in the old one with renormalized variables. The operator $\mathcal{D}_{N}$ is therefore defined explicitly as an operator acting on the paths internal to four-site cells. It selects for each $N$ just relaxation paths that consist of $N$ connected noncontemporary relaxation events that leave the cell without unstable sites. In a mathematical form it reads as

$$
\mathcal{D}_{N}=\prod_{i \in\left\{\alpha^{\prime}\right\}}\left(1-\delta_{2, s_{i}}\right) \prod_{J=0}^{N-1} \sum_{m=1}^{4} \delta\left(m-\sum_{i \in\left\{\alpha_{J}\right\}} \delta_{2, s_{i}}\right),
$$

where $\alpha_{J}$ 's are the intermediate cell configurations during the dynamical evolution and $\Sigma_{i \in\left\{\alpha_{J}\right\}}$ denotes the sum over all the sites in the cells. In the above expression, each $\delta$ function acts on a different intermediate cell, eliminating those paths that do not have activity at each dynamical step. Furthermore, the operator ensures that in the cell $\alpha^{\prime}$ ( $N$ th step) no activity is present; i.e., the process has stopped. Finally, we have to write the equation that gives the time scaling factor from the total average over contributing processes to the renormalized matrix element $\left\langle 0\left|T^{\prime}\right| 2\right\rangle$,

$$
g\left(p_{n}\right)=\frac{1}{\mathcal{N}} \sum_{\alpha} W_{\alpha}(\langle\rho\rangle) \sum_{\alpha^{\prime}} \sum_{N} N \mathcal{D}_{N}\left\langle\alpha^{\prime}\left|T^{N}\left(p_{n^{\prime}}\right)\right| \alpha\right\rangle,
$$

where we used the DDRG scheme to explicitly get the stationary weights, and $\mathcal{N}$ is an opportune normalization factor. The above relations will provide the consistent rescaling of time by requiring that $b^{z}=g\left(p_{n}^{*}\right)$, from which it is possible to calculate the dynamical critical exponent.

\section{MINIMAL PROLIFERATION CALCULATIONS AND RESULTS}

The explicit evaluation of the recursion relations depends on the choice of the spanning condition. In the following, the scheme used considers only connected paths that span the

cell from left to right or from top to bottom. This spanning rule implies that only paths extending over the size of coarse grained length scale contribute to the renormalized dynamics, and it ensures the connectivity of the avalanche in the renormalization procedure.

An example of such a path is shown in Fig. 1. In this case $\alpha=2$ and the path shown refers to the probability that the unstable subcell will relax towards the other critical subcell [Fig. 1(b)]. This occurs with probability $(1 / 4) p_{1}$. At this point we consider the probability that the next relaxation event at the fine scale will involve two neighboring sites, one inside and the other outside the original cell of size $b=2$ [Fig. 1(c)]. This occurs with probability $(2 / 3) p_{2}$. This series of relaxation processes contributes to the term $\mathcal{D}_{2}\left\langle\alpha^{\prime}\left|T^{2}\left(p_{n^{\prime}}\right)\right| \alpha=2\right\rangle_{1}$ that characterizes the relaxation pro-

(a)

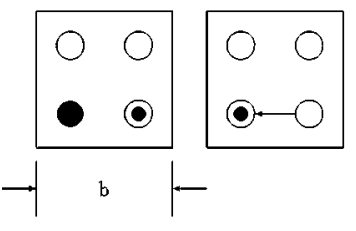

FIG. 1. Example of the renormalization scheme for the relaxation dynamics. For details see the text. 
cesses at the coarse grained scale. By summing over all the paths that lead to a $p_{1}^{\prime}$ process, one obtains for $\alpha=2$

$$
\begin{aligned}
\sum_{\alpha^{\prime}} \sum_{N} \mathcal{D}_{N}\left\langle\alpha^{\prime}\left|T^{N}\left(p_{n^{\prime}}\right)\right| \alpha=2\right\rangle_{1}= & \left(\frac{1}{4} p_{1}+\frac{1}{6} p_{2}\right)\left(\frac{1}{2} p_{1}+\frac{2}{3} p_{2} \frac{1}{2} p_{3}\right)+\left(\frac{1}{6} p_{2}+\frac{1}{4} p_{3}\right)\left(\frac{1}{2} p_{1}+\frac{1}{6} p_{2}\right) \\
& +\left(\frac{1}{6} p_{2}+\frac{1}{4} p_{3}\right)\left(\frac{1}{6} p_{2}+\frac{1}{4} p_{3}\right)\left(\frac{3}{4} p_{1}+\frac{1}{2} p_{2} \frac{1}{4} p_{3}\right) .
\end{aligned}
$$

In a similar way one can also write the expression for the complete recursion relations. Proliferation effects due to multiple relaxations of the same site and sites becoming critical during the dynamical process are not considered in this scheme. However, the complete polynomials for $p_{n}^{\prime}$ involve more than 200 terms, and we developed a generating function that allows for their systematic calculation.

The generating function allows us to find the form of the renormalized operator $T^{\prime}$, without writing down the explicit form of all relaxation paths in the coarse grained cell. The basic idea of this method is to renormalize the function that describes all relaxation paths at once, rather than the probabilities of separate relaxation paths, by using the branching structure of avalanches.

To describe in detail the branching process underlying the large scale behavior of the sandpile model, we consider the generating function

$$
\begin{aligned}
\sigma(N, E, S, W)= & p_{0}+\frac{p_{1}}{4}(N+E+S+W) \\
& +\frac{p_{2}}{6}(N E+N S+N W+E S+E W+S W) \\
& +\frac{p_{3}}{4}(N E S+N E W+N S W+E S W) \\
& +p_{4} N E S W
\end{aligned}
$$

where the symbols $N, E, S$, and $W$ correspond to the north, east, south, and west directions on the square lattice, respectively. The coefficient in each term of this polynomial gives the probability for the process to go in the corresponding directions. The generating function takes into account all possible relaxation processes in the cell. It is easy to check directly that this function has the following properties:

(i) If the argument corresponding to any direction is replaced by zero, the function counts the relaxation processes that do not send energy to this direction [Fig. 2(b)].

(ii) If the argument corresponding to any direction is replaced by a unit, the function counts the relaxation processes whether or not the energy is transferred in that direction [Fig. $2(\mathrm{c})]$.

(iii) The generating function is normalized so that $\sigma(1,1,1,1)=1$.

If there is a critical cell near the relaxing one, the outgoing energy can initiate the relaxation of the cell. It is easy to see that we can replace the argument corresponding to this direction by another generating function corresponding to the relaxation of the second cell. Finally, we obtain the generating function of this two-step relaxation process. For ex-

ample, the function $\sigma\left(N_{1}, \sigma\left(N_{2}, E_{2}, S_{2}, 1\right), S_{1}, W_{1}\right)$ describes the processes where cell 1 relaxes first. Then, if the energy goes to the direction $N_{1}$ it initiates the relaxation of cell 2 [Fig. 2(d)]. The symbols $N_{i}, E_{i}, S_{i}, W_{i}$ denote the directions outgoing from cell $i$.

Using these properties we can write down the generating function $\Sigma_{\alpha}$, counting the relaxation processes in the block that consists of four cells for different $\alpha$. To this end, we must take into account only the processes that match the spanning condition. Therefore, it is necessary to eliminate all processes, in which only one cell relaxes. As the coefficients of the polynomial $\Sigma_{\alpha}$ mean probabilities, they should finally be normalized by the condition

$$
\Sigma_{\alpha}(1, \ldots, 1)=1 \text {. }
$$

The generating function corresponding to the relaxation processes inside the block with $\alpha=2$ is

$$
\begin{aligned}
\Sigma_{2}= & \left\{\sigma\left(\sigma\left(N_{2}, 1,1, W_{2}\right), 1, S_{1}, W_{1}\right)-\sigma\left(0,1, S_{1}, W_{1}\right)\right\} \\
& +\left\{\sigma\left(N_{2}, 1, \sigma\left(1,1, S_{1}, W_{1}\right), W_{2}\right)-\sigma\left(N_{2}, 1,0, W_{2}\right)\right\} \\
& + \text { c.p. } / Z_{2},
\end{aligned}
$$

(a)

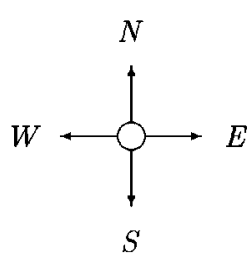

$\sigma(N, E, S, W)$

(b)
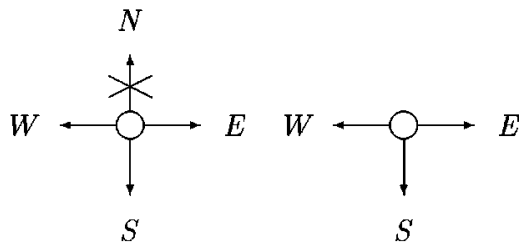

$S$

(d)

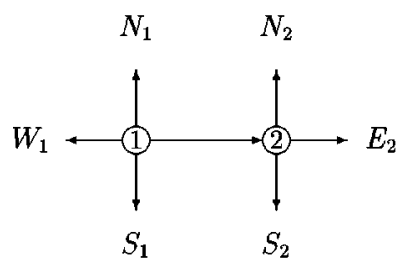

$$
\sigma\left(N_{1}, \sigma\left(N_{2}, E_{2}, S_{2}, 1\right), S_{1}, W_{1}\right)
$$

FIG. 2. Examples of generating functions' application. 
where $Z_{2}$ is a normalization factor chosen so that $\Sigma_{2}(1, \ldots, 1)=1$. To write this function we start from the left down cell and define the arguments of the $\sigma$ function corresponding to the toppling of this cell. Because the process definitely spans the block, the left up cell should topple and we write another $\sigma$ function instead of the symbol $N_{1}$. By going eastward the process will terminate inside the block and this branch of the toppling process cannot affect the neighboring blocks. Hence, we should write the number 1 instead of the symbol $E_{1}$. The other symbols $S_{1}$ and $W_{1}$ correspond to the branches of the relaxation process that go immediately out of the initial block of cells. To consider only the processes that span the block, we must subtract the $\sigma$ function that describes processes that do not send the energy from the first critical cell to the second one. Then, we add analogous $\sigma$ functions for the processes starting from the relaxation of the left upper cell. The term c.p. denotes all possible cyclic permutations of the critical cells inside the block. Analogously, we can write $\sigma$ functions of all other types of blocks.

To obtain the complete generating function $\Sigma$ for the block of four cells, we should sum up all $\Sigma_{\alpha}$ functions with the weights of blocks and normalize the result

$$
\Sigma=\frac{1}{Z} \sum_{\alpha} W_{\alpha} \Sigma_{\alpha} .
$$

Now, to transform the $\Sigma$ function from the block of four cells at the scale $b^{k}$ into a larger cell at the next scale $b^{k+1}$, we replace the directions $N_{1}, N_{2}, \ldots$ that are outgoing from the initial block by the new arguments corresponding to the directions $N, \ldots$ that are outgoing from the new renormalized cell. In other words, two bonds that connect the neighboring blocks are coupled to the only bond on the lattice at the next scale, as is shown in Fig. 3. Eventually, we obtain the following generating function:

$$
\Sigma(N, E, S, W)=\frac{W_{2} \Sigma_{2}+W_{3} \Sigma_{3}+W_{4} \Sigma_{4}}{Z},
$$

where

$$
\begin{aligned}
\Sigma_{2}= & (\sigma(\sigma(N, 1,1, W), 1, S, W)-\sigma(0,1, S, W)) \\
& +(\sigma(N, 1, \sigma(1,1, S, W), W)-\sigma(N, 1,0, W))+\text { c.p. } / Z_{2},
\end{aligned}
$$

$$
\begin{aligned}
\Sigma_{3}(N, E, S, W) & \\
= & (\sigma(\sigma(N, \sigma(N, E, 1,1), 1, W), 1, S, W)-\sigma(0,1, S, W) \\
& +\sigma(\sigma(N, 1,1, W), \sigma(1, E, S, 1), S, W)-\sigma(0,0, S, W) \\
& +\sigma(1, \sigma(\sigma(N, E, 1,1), E, S, 1), S, W)-\sigma(1,0, S, W) \\
& + \text { c.p. }) / Z_{3},
\end{aligned}
$$

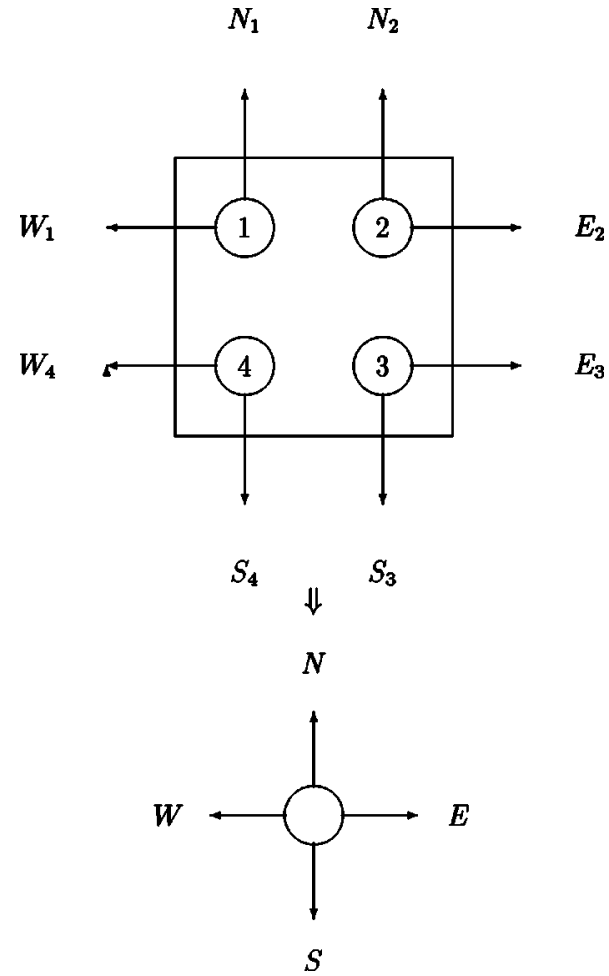

FIG. 3. Lattice renormalization by grouping two bonds connecting neighboring blocks into a single bond of the rescaled lattice. $N_{1}, N_{2} \rightarrow N, E_{2}, E_{3} \rightarrow S, S_{3}, S_{4} \rightarrow W, W_{4}, W_{1} \rightarrow E$.

$$
\begin{aligned}
\Sigma_{4}(N, E, S, W) \\
=(\sigma(\sigma(N, \sigma(N, E, \sigma(1, E, S, 1), 1), 1, W), 0, S, W) \\
+\sigma(0, \sigma(\sigma(N, E, 1, \sigma(N, 1,1, W)), E, S, 1), S, W) \\
-2 \sigma(0,0, S, W)+(\sigma(1,1, S, W)-\sigma(1,0, S, W) \\
-\sigma(0,1, S, W)+\sigma(0,0, S, W))(\sigma(N, 0,1, W) \sigma(0, E, S, 1) \\
+\sigma(N, E, 1,1)(\sigma(N, 0,1, W)(\sigma(1, E, S, 1) \\
-\sigma(0, E, S, 1))+\sigma(0, E, S, 1)(\sigma(N, 1,1, W) \\
-\sigma(N, 0,1, W))+(\sigma(1, E, S, 1) \\
-\sigma(0, E, S, 1))(\sigma(N, 1,1, W) \\
-\sigma(N, 0,1, W)))+ \text { c.p. }) / Z_{4} .
\end{aligned}
$$

Here, $Z_{i}$ and $Z$ are the normalization factors and c.p. denote the expressions obtained from the previous polynomial by all possible cyclic permutations of its arguments. This generating function is the polynomial that contains only the first and second powers of its arguments. The last terms correspond to the processes when two energy portions are transferred from the initial block to the neighboring block by the two paths. However, according to the RG strategy, these processes should be considered as the transfer of the coarse grained energy portion at the larger scale. Therefore, all second powers of the arguments should be replaced by the first ones. The result obtained is the generating function describing the relaxation of renormalized cells. It depends on the same products of its arguments as the generating function for initial cells, but the coefficients of this polynomial are different and 
TABLE I. The fixed point probabilities for the energy transfer from the relaxing cell, including the probability $p_{0}$.

\begin{tabular}{cccccc}
\hline \hline & $p_{0}^{*}$ & $p_{1}^{*}$ & $p_{2}^{*}$ & $p_{3}^{*}$ & $p_{4}^{*}$ \\
\hline$\rho^{*}=0.595$ & 0.091 & 0.345 & 0.379 & 0.161 & 0.024 \\
\hline \hline
\end{tabular}

equal to the probabilities of relaxation processes that send energy to the given direction at the new scale. Taking these coefficients, we obtain the sought recursion relations that link the parameters of the cell at the scale $b^{k}$ with the same parameters at the scale $b^{k+1}$,

$$
\vec{P}(k+1)=\vec{f}(\vec{P}(k), \rho(k)) .
$$

The above set of RG equations supplemented with the driving condition

$$
\left\langle\rho^{k+1}\right\rangle=\frac{1}{\sum_{n} n p_{n}^{k+1}}
$$

define completely the DDRG recursion relations for sandpile models. Given this scheme, the flow diagram and the relative fixed point in the parameters space $(\rho, \vec{P})$ can be studied. We consider here the calculation scheme implemented with $\vec{P}$ $=\left(p_{0}, p_{1}, p_{2}, p_{3}, p_{4}\right)$. Despite the enlargement of the phase space by including the proliferation characterized by the probability $p_{0}$, the flow in the phase space is very similar to those obtained in Ref. [11], where this parameter was not considered. A single attractive fixed point is obtained and the numerical value of this fixed point is very close to that obtained in the approach of Ref. [11]. The complete attractiveness of the fixed point corresponds to the lack of relevant scaling field, i.e., control parameter. This must be the case, because we implement our RG scheme in the infinite time scale separation limit. In this limit the driving field (addition of sand grains or energy) is infinitesimally small; i.e., it is tuned to its critical value. This implies that the relevant scaling fields are constrained to their critical values. In other words we are restricting the study of the system on its critical surface. In Table I we report the results obtained within this calculation scheme. The single fixed point is the signature of a single universality class for all the nondirected sandpile models. The identification of universality classes in sandpile models is a longstanding and still unresolved issue. The results obtained by numerical simulations are unclear. Early large scale numerical simulations $[20,21]$ show that avalanche distributions are described by the same exponents in both stochastic and Abelian sandpile. Later, these results have been questioned in more recent papers [23,33], where a new classification in which Abelian and stochastic models belong to different universality classes is proposed. The present approach supports the existence of such a single universality class; however, it cannot provide a definitive settling of the issue. Mainly, it depends on the fact that the method neglects proliferations such as the possibility of multiple topplings, which could be relevant for the identification of different universality classes.
The avalanche exponent $\tau$ can be obtained directly from the fixed point parameters. By using the discrete length scale $b^{(k)}=2^{k}$ and the avalanche distribution in the form $P(r) d r$ $\approx r^{(1-2 \tau)} d r$, we can define the probability that the relaxation process will span the cell of size $b^{(k)}$ and die at the neighboring cells not extending over the scale $b^{(k+1)}$,

$$
K=\int_{b^{(k-1)}}^{b^{(k)}} P(r) d r / \int_{b^{(k-1)}}^{\infty} P(r) d r=1-2^{2(1-\tau)} .
$$

Asymptotically $(k \rightarrow \infty)$ we can express $K$ in terms of fixed point parameters $\rho^{*}$ and $p_{i}^{*}$ in the following way:

$$
\begin{aligned}
K= & p_{0}^{*}+p_{1}^{*}\left(1-\rho^{*}\right)+p_{2}^{*}\left(1-\rho^{*}\right)^{2}+p_{3}^{*}\left(1-\rho^{*}\right)^{3}+p_{4}^{*}(1 \\
& \left.-\rho^{*}\right)^{4}
\end{aligned}
$$

This equation gives the total probability that a relaxation process will occur without triggering other sites, and therefore it does not extend on length scales larger than that of a single cell. Using these two expressions, Eqs. (32) and (33), the exponent $\tau$ is given by the formula

$$
\tau=1-\frac{1}{2} \frac{\ln (1-K)}{\ln 2}=1.262,
$$

in excellent agreement with the proposed value $\tau=5 / 4$ [30] and large scale numerical simulations [20,21,23]. The obtained value is also in good agreement with the value obtained in the calculation of Ref. [11], showing the robustness of the method with respect to different proliferation schemes. In order to overcome some of the approximations considered so far, we will present in the next section an improved scheme that takes into account a wider set of dynamical parameters. This scheme allows us to also study the critical behavior at the boundary of the system.

\section{EXTENDED KINETIC EQUATION SCHEME}

In this section we treat more explicitly the dynamics of the original sandpile model. Considering the evolution of stable cells, we can take into account some of the processes that were neglected in the scheme discussed in the previous section.

To keep the connection with the original formulation of the sandpile model, we will characterize the static properties of a cell by four quantities,

$\vec{N}(k)=\left(n_{A}, n_{B}, n_{C}, n_{D}\right), \quad n_{A}+n_{B}+n_{C}+n_{D}=1$,

which are nothing but the probabilities of a cell behaving like a site on the initial lattice with a height $1,2,3$, or 4, respectively, in the coarse grained dynamics; i.e., the addition of a "coarse grained particle" to the cell transforms it into the next one in the alphabet. For example, the cell $B$ characterized by the vector $(0,1,0,0)$ will be transformed into the cell $C$ with the vector $(0,0,1,0)$. The last variable $n_{D}$ is the probability of the cell behaving like a critical one in a sense that the addition of a "coarse grained particle" to the cell induces relaxations into some neighboring cells or, in 
other words, subrelaxation processes on a minimal scale span the cell and transfer energy to some of its neighbors.

As we stressed in the previous sections, independently of the dynamics of the model at the minimal scale, each critical cell is characterized by the vector

$$
\vec{P}(k)=\left(p_{1}, p_{2}, p_{3}, p_{4}\right), \quad p_{1}+p_{2}+p_{3}+p_{4}=1,
$$

which gives the probabilities of the energy going to 1, 2, 3, or 4 neighboring cells after the relaxation of the critical cell. Here, because we have already enlarged the phase space by introducing the densities $n_{\alpha}$, we do not include $p_{0}$ in the calculation scheme.

In this framework the coarse grained dynamics of the sandpile model can be represented as the following branching process on the sublattice $\mathcal{L}_{b}$ :

$$
\begin{aligned}
& A+\varphi \rightarrow B, \\
& B+\varphi \rightarrow C, \\
& C+\varphi \rightarrow D, \\
& D+\varphi \rightarrow\left\{\begin{array}{l}
p_{1}: D+\tilde{\varphi} \\
p_{2}: C+2 \tilde{\varphi} \\
p_{3}: B+3 \tilde{\varphi} \\
p_{4}: A+4 \tilde{\varphi} .
\end{array}\right.
\end{aligned}
$$

Here, $\varphi$ and $\tilde{\varphi}$ denote the "coarse grained particles" obtained by the cell and the particles transferred to the neighboring cells, respectively.

These processes can be formally reinterpreted as an irreversible chemical reaction that takes place at each cell of the sublattice $\mathcal{L}_{b}$. Now the coarse grained variables $n_{A}, n_{B}, n_{C}, n_{D}$, and $n_{\varphi}$ denote the concentrations of the respective species $A, B, C, D$, and $\varphi$. Following standard prescriptions of the chemical physics, we can write down kinetic equations corresponding to this scheme of chemical reactions,

$$
\begin{aligned}
& \dot{n}_{A}=n_{\varphi}\left(p_{4} n_{D}-n_{A}\right), \\
& \dot{n}_{B}=n_{\varphi}\left(p_{3} n_{D}+n_{A}-n_{B}\right), \\
& \dot{n}_{C}=n_{\varphi}\left(p_{2} n_{D}+n_{B}-n_{C}\right), \\
& \dot{n}_{D}=n_{\varphi}\left(p_{1} n_{D}+n_{C}-n_{D}\right), \\
& \dot{n}_{\varphi}=n_{\varphi}\left(\bar{p} n_{D}-1\right)+\bar{p} \nu \nabla^{2}\left(n_{\varphi} n_{D}\right)+\eta(\mathbf{r}, t),
\end{aligned}
$$

where $\bar{p}=p_{1}+2 p_{2}+3 p_{3}+4 p_{4}$ is equal to the average number of particles leaving the cell on toppling and $\mathbf{r}$ is the position vector of the cell in the two-dimensional space. The noise term $\eta(\mathbf{r}, t)$, being non-negative, mimics the random addition of particles to the system. The diffusion term $\nabla^{2}\left(n_{\varphi} n_{D}\right)$ describes the transfer of particles into the neighboring cells, and the diffusion coefficient $\nu$ for the discrete Laplacian on the square lattice is equal to $1 / 4$.
The only mobile species in this scheme of reactions is $\varphi$, and it is the field $n_{\varphi}$, which describes the dynamics of avalanches. When it is equal to zero, all toppling processes die. Then, due to the noise term $\eta(\mathbf{r}, t)$, particles are added randomly into the system initiating a branching process directed to the open boundary of the system. This process mutates species in the cells it has visited and topples the critical ones. Finally, the system will reach the steady state where the probability that the activity will die is on average balanced by the probability that the activity will branch. Thus, the chain reaction maintains this stationary state and all further avalanches cannot change the concentrations of species $A, B, C$, and $D$. Therefore, the steady state is characterized by the conditions that

$$
\dot{n}_{A}=\dot{n}_{B}=\dot{n}_{C}=\dot{n}_{D}=0
$$

and Eqs. (38)-(41) lead to the following relationships between concentrations of species $\vec{N}(k)$ at the stationary state and branching probabilities $\vec{P}(k)$,

$$
\begin{aligned}
& n_{A}^{*}=p_{4} / \bar{p}, \\
& n_{B}^{*}=\left(p_{3}+p_{4}\right) / \bar{p}, \\
& n_{C}^{*}=\left(p_{2}+p_{3}+p_{4}\right) / \bar{p}, \\
& n_{D}^{*}=\left(p_{1}+p_{2}+p_{3}+p_{4}\right) / \bar{p}=1 / \bar{p} .
\end{aligned}
$$

The relation (47) between the probability $n_{D}^{*}$ and branching probabilities $\vec{P}(k)$ can also be derived from the assumption that at the stationary state the flow of particles in a cell was on average balanced by the flow of particles out of the cell.

Thus, we have found the driving conditions for the sandpile models. Using them we can link the statistic weights of any static configurations of a cell with the dynamic parameters. Now, we can realize the renormalization procedure described previously. To this end, we must consider all types of blocks of four cells, whose relaxation matches the spanning condition. Such blocks and some of their relaxation schemes are shown in Fig. 4. While the previous scheme deals only with the cells being critical before the relaxation of the block, this one allows us to consider the cells becoming critical during the relaxation [Fig. 4(c)].

To obtain the recursion relations by the method presented in the preceding section, it is necessary to calculate the statistical weights of all configurations considered. The statistical weight of the block is given by the product of probabilities $n_{i}$ for all cells in the block, multiplied by the number of different blocks with the same relaxation schemes. Thus, the following weights must be ascribed to the blocks shown in Fig. 4:

$$
\begin{aligned}
& W_{a}=4 n_{D}^{2}\left(n_{A}+n_{B}+n_{C}\right)^{2}, \\
& W_{b}=4 n_{D}^{3}\left(n_{A}+n_{B}\right), \\
& W_{c}=4 n_{D}^{3} n_{C}, \\
& W_{d}=n_{D}^{4} .
\end{aligned}
$$


(a)

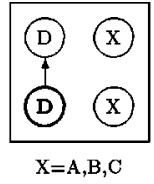

(b)
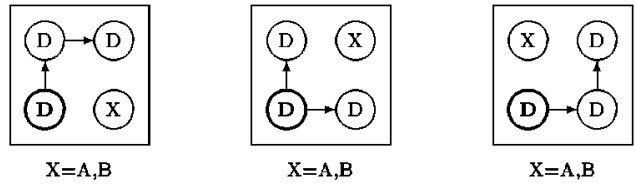

(c)
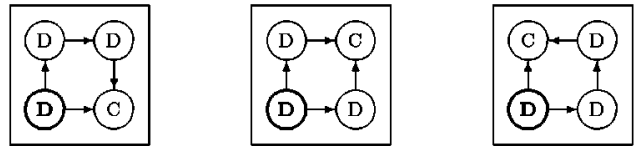

(d)

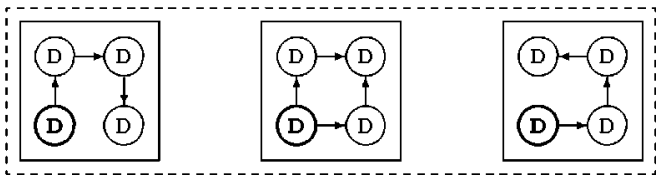

FIG. 4. We show the four different elementary blocks in the chemical reaction model and some relaxation schemes spanning them. The other schemes can be obtained from these figures by rotations. It is convenient for calculations to subdivide the relaxation process in block (d) into three processes, as shown in the dashed box.

Expressing them through the probabilities $\vec{P}$, by using driving conditions (44)-(47), we obtain the complete system of renormalization equations:

$$
\vec{P}(k+1)=\vec{f}(\vec{P}(k)) .
$$

Given this set of RG transformations we can study how the system evolves under successive doublings of length scale. The final result is independent of the initial conditions $n_{i}$ and $p_{i}$ at the minimal scale. Also in this case the fixed point is attractive in the whole phase space and the system evolves spontaneously toward the fixed point values $p_{i}^{*}$ and $n_{i}^{*}$ shown in Tables II and III. These results can be compared with the exact ones obtained for the sandpile [26]. The exact height probabilities for the sandpile are reported in Table III and compared with our RG results. In order to calculate the avalanche exponent we can use Eq. (32) by expressing $K$ in terms of the fixed point parameters in the following way:

$$
\begin{aligned}
K= & p_{1}^{*}\left(1-n_{D}^{*}\right)+p_{2}^{*}\left(1-n_{D}^{*}\right)^{2}+p_{3}^{*}\left(1-n_{D}^{*}\right)^{3} \\
& +p_{4}^{*}\left(1-n_{D}^{*}\right)^{4} .
\end{aligned}
$$

TABLE II. Relaxation probabilities in the extended RG scheme.

\begin{tabular}{lcccc}
\hline \hline & $p_{1}^{*}$ & $p_{2}^{*}$ & $p_{3}^{*}$ & $p_{4}^{*}$ \\
\hline Bulk & 0.295 & 0.435 & 0.229 & 0.0414 \\
\hline Open & 0.142 & 0.417 & 0.351 & 0.0899 \\
\hline Closed & 0.526 & 0.394 & 0.0799 & \\
\hline \hline
\end{tabular}

TABLE III. Height probabilities in the stationary state.

\begin{tabular}{lccccc}
\hline \hline & & $n_{A}$ & $n_{B}$ & $n_{C}$ & $n_{D}$ \\
\hline Bulk & RG & 0.0205 & 0.134 & 0.349 & 0.496 \\
& Exact [26] & 0.0736 & 0.174 & 0.306 & 0.446 \\
\hline Open & RG & 0.0377 & 0.184 & 0.359 & 0.419 \\
boundary & Exact [27] & 0.104 & 0.217 & 0.316 & 0.363 \\
\hline Closed & RG & 0.0514 & 0.305 & 0.643 & \\
boundary & Exact [27] & 0.113 & 0.318 & 0.568 & \\
\hline \hline
\end{tabular}

More generally the probability (53) should be represented via $\sigma$ function

$K=\sigma^{*}\left(1-n_{D}^{*}(N), 1-n_{D}^{*}(E), 1-n_{D}^{*}(S), 1-n_{D}^{*}(W)\right)$,

where $n_{D}(N), n_{D}(E), n_{D}(S)$, and $n_{D}(W)$ are the concentrations of critical cells at the nearest neighbors. By using the fixed point values we finally obtain $\tau=1.248$, which again is in very good agreement with the proposed value 5/4 [30] and numerical simulations $[20,21,23]$. Also, in this case the scheme results in a single universality class for the sandpile models. In the extended scheme we include part of the proliferations by allowing different heights, and taking into account that some of the sites becomes critical during the relaxation event. We do not, however, consider multiple relaxation of the same sites during the spanning time, nor do we allow a renormalization of the energy transfer $(\delta E)$. These parameters could be important in the case of the Manna model [20], as pointed out in Ref. [33]. Work is in progress to extend the present DDRG scheme in order to include further proliferations also.

\section{BOUNDARY CRITICAL PROPERTIES}

Since the critical properties of the sandpile model are quite similar to those of second order phase transitions, we proceed here along the same lines followed in the study of equilibrium critical phenomena. In particular, we also determine the surface critical exponents, which in general differ from the bulk ones. This is of special importance in the twodimensional case where conformal field theory connects surface and bulk properties of the model [34].

\section{A. Open boundary}

The fact that the boundary is open means that after the relaxation of the boundary site the energy can leave the system. We consider the critical energy of sites at the open boundary to be $E_{c}=4$. It is more convenient to consider the boundary lying along the diagonal of the lattice and construct the renormalized cell in the following way. We consider the block of cells $2 \times 2$ that contains one bulk, two boundary, and one external cells, as shown in Fig. 5. Because the critical properties of the model at large scales do not depend on the local structure of the lattice, the results obtained should not depend on the specific choice of the boundary. To describe the boundary cells at an arbitrary scale, we introduce the vectors 


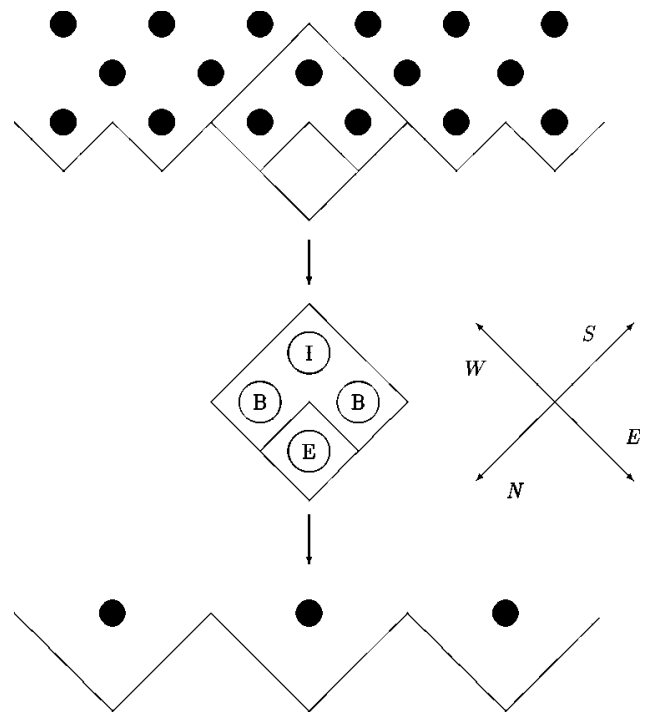

FIG. 5. Cell-to-site transformation procedure at the lattice open boundary. One internal cell (I), two boundary cells (B), and one auxiliary external cell (E) build the boundary cell at the coarse grained scale.

$\vec{N}^{o}(k)=\left(n_{A}^{o}, n_{B}^{o}, n_{C}^{o}, n_{D}^{o}\right), \quad n_{A}^{o}+n_{B}^{o}+n_{C}^{o}+n_{D}^{o}=1$,

$\vec{P}^{o}(k)=\left(p_{1}^{o}, p_{2}^{o}, p_{3}^{o}, p_{4}^{o}\right), \quad p_{1}^{o}+p_{2}^{o}+p_{3}^{o}+p_{4}^{o}=1$,

which have the same meaning as in the preceding section. The kinetic equations and feedback relations coincide with the bulk ones. Thus, it is only necessary to find the correct form of recursion relations. To write the generating function for the boundary block, we can use again the generating functions describing the relaxations of bulk and boundary cells. Also we have to introduce a special generating function corresponding to the relaxation of the unphysical external cell. Since there are not processes transferring energy from the external half-plane of the lattice to the internal one, we require that the external cell immediately transfer the energy outside the lattice. Thus, we provide conservation of the flow of energy through the boundary in the scale transformation. For the block shown in Fig. 5 the generating function of relaxation of the external cell has the simple form

$$
\sigma^{\text {out }}=\frac{N+E}{2} .
$$

Statistical weights of the static boundary configurations are given by the product of the probabilities of one bulk and two boundary cells. Now, the coefficients of the generating function of the renormalized cell gives us the recursion relations

$$
\vec{P}^{o}(k+1)=\vec{f}_{o}\left(\vec{P}^{o}(k), \vec{P}(k)\right) .
$$

Together with the bulk recursion relations of Eq. (52), Eq. (58) represents the complete system of the renormalization equations for the case of the open boundary. This system also has only one fixed point. The obtained fixed point parameters are given in Tables II and III. The comparison of fixed point height probabilities with the exact values obtained for ASMs [27] shows rather good agreement.
To calculate the boundary critical exponent we should use the generating function describing the relaxation of the boundary cell in the fixed point of RG flow. It is given by Eq. (32), where

$$
K=\sigma_{o}^{*}\left(1,1,1-n_{D}^{o}, 1-n_{D}^{o}\right) .
$$

This indicates that the toppling of the coarse grained boundary cell should stop in two neighboring internal cells. The result $\tau_{o}=1.486$ is very close to $\tau_{o}=\frac{3}{2}$ calculated exactly in [28] for the open boundary of an ASM. Such a good agreement is probably to be ascribed to the fact that in the boundary avalanche in an ASM each site topples only once [29]. Therefore a renormalization scheme that does not take into account multiple topplings gives the most realistic results near the open boundary.

\section{B. Closed boundary}

Let us consider the sandpile model on the half-plane. The edge of this half-plane is the closed boundary directed along one of the lattice axes. Each boundary site has three neighboring sites. Two of them also belong to the boundary. The fact that the boundary is closed means that after the relaxation of a boundary site energy does not leave the lattice, being distributed among the neighboring sites. Since the energy can leave the boundary site in only three directions, it is quite natural to take the critical energy of a boundary site $E_{c}=3$.

In order to follow the RG strategy, we again perform the site-to-cell transformation, replacing the block of four cells by a single cell at the larger scale. The cells at an arbitrary scale can be considered as either boundary or bulk ones. While the former consist only of bulk cells at the smaller scale, the latter include both the bulk and the boundary cells of smaller size. The renormalization of the bulk cell is described by the system of RG equations obtained in the preceding section. Let us introduce the description for the dynamics of boundary cells at an arbitrary scale. The static states of a boundary cell can be represented by three symbols $A, B, C$, which correspond to energy values $E=1,2,3$ of boundary sites on the initial lattice. The addition of energy transforms the cell from state $A$ into state $B$ and the cell from state $B$ into state $C$. The cell in state $C$ is critical. The addition of energy initiates its relaxation when the cell turns into states $A$ or $B$ or remains in state $C$, sending the energy to three, two, or one neighboring cell, respectively. The probabilities for the cell on the closed boundary to be in one of the three states are given by the vector

$$
\vec{N}^{c}(k)=\left(n_{A}^{c}, n_{B}^{c}, n_{C}^{c}\right), \quad n_{A}^{c}+n_{B}^{c}+n_{C}^{c}=1 .
$$

In the same way, the probabilities for the energy to be transferred in one, two, or three directions are given by the vector

$$
\vec{P}^{c}(k)=\left(p_{1}^{c}, p_{2}^{c}, p_{3}^{c}\right), \quad p_{1}^{c}+p_{2}^{c}+p_{3}^{c}=1 .
$$

The relaxation process at the boundary cell can be represented as follows: 


$$
\begin{aligned}
& A+\varphi \rightarrow B, \\
& B+\varphi \rightarrow C, \\
& C+\varphi \rightarrow\left\{\begin{array}{l}
p_{1}^{c}: C+1 \tilde{\varphi} \\
p_{2}^{c}: B+2 \tilde{\varphi} \\
p_{3}^{c}: A+3 \tilde{\varphi},
\end{array}\right.
\end{aligned}
$$

where $\varphi$ and $\tilde{\varphi}$ are the energy obtained by the cell and transferred to the neighboring cell, respectively. Hence, we can write the following kinetic equations for the energy transfer:

$$
\begin{aligned}
& n_{A}^{{ }^{c}}=n_{\varphi}\left(p_{3}^{c} \quad n_{C}^{c}-n_{A}^{c}\right), \\
& n_{B}^{c}=n_{\varphi}\left(p_{2}^{c} n_{C}^{c}+n_{A}^{c}-n_{B}^{c}\right), \\
& n_{C}^{c}=n_{\varphi}\left(p_{1}^{c} n_{C}^{c}+n_{B}^{c}-n_{C}^{c}\right), \\
& n_{\varphi}^{c}=n_{\varphi}\left(\bar{p} c n_{D}-1\right)+\bar{p} c \nu \nabla^{2}\left(n_{\varphi} n_{C}^{c}\right)+\eta(\mathbf{r}, t) .
\end{aligned}
$$

Here, the discrete Laplacian $\Delta$ must be understood with the Neumann boundary conditions. The steady state corresponds to the conditions, $\dot{\vec{N}}^{c}=0$. This leads us to the following driving conditions for the closed boundary:

$$
\begin{aligned}
& n_{A}^{c}=p_{3}^{c} / \bar{p}, \\
& n_{B}^{c}=\left(p_{2}^{c}+p_{3}^{c}\right) / \bar{p}^{c}, \\
& n_{C}^{c}=\left(p_{1}^{c}+p_{2}^{c}+p_{3}^{c}\right) / \bar{p}^{c}=1 / \bar{p}^{c} .
\end{aligned}
$$

To perform the standard renormalization procedure described above and find the recursion relations, the generating function method can be employed. To this end, we introduce the generating function for the relaxation of a cell on the closed boundary as the following polynomial:

$$
\begin{aligned}
\sigma_{c}(N, E, W)= & \frac{p_{1}^{c}}{3}(N+E+W)+\frac{p_{2}^{c}}{3}(N E+N W+E W) \\
& +p_{3}^{c} N E W .
\end{aligned}
$$

The general idea of the generating function for the block of four cells is the same as in the bulk case. The difference is that the generating functions for boundary blocks oriented differently with respect to the boundary should be calculated separately and cannot be obtained by simple cyclic permutations of the arguments. Finally, applying the renormalization procedure with the use of the feedback relations (66)-(68), we obtain the recursion relations

$$
\vec{P}^{c}(k+1)=\vec{f}_{c}\left(\vec{P}^{c}(k), \vec{P}(k)\right),
$$

where $\vec{P}(k)$ matches the bulk recursion relations of Eq. (52). The obtained height probabilities (Table III) are in good agreement with those calculated exactly in the case of an ASM with closed boundary. To calculate the critical
TABLE IV. Critical exponents $\tau, \tau_{o}$, and $\tau_{c}$ for the avalanche size distribution in the bulk, open, and closed boundaries, respectively.

\begin{tabular}{lccc}
\hline \hline & $\tau$ & $\tau_{o}$ & $\tau_{c}$ \\
\hline RG & 1.248 & 1.486 & 1.239 \\
\hline Exact & $1.25[30]$ & $1.5[28]$ & $?$ \\
\hline \hline
\end{tabular}

exponent $\tau_{c}$ describing the distribution of avalanches near a closed boundary, we use Eq. (32), expressing $K$ through the fixed point generating function of the boundary cell:

$$
K=\sigma_{c}^{*}\left(1-n_{C}^{c}, 1-n_{D}, 1-n_{C}^{c}\right) .
$$

The critical exponent becomes $\tau_{c}=1.239$. The correction to the bulk critical exponent due to the half-plane geometry has not, to our knowledge, been presented before. The obtained value is slightly different from the one obtained for the bulk exponent. The approximate nature of the method, however, does not allow us to determine if the two exponents are actually different or if this is just due to the truncation and proliferation scheme used.

\section{DISCUSSION AND CONCLUSIONS}

In this paper we have presented the detailed application of the DDRG to the sandpile model. We have concentrated on the Bak-Tang-Wiesenfeld model, which we have studied using schemes of increasing complexity. In the simple scheme the sites are subdivided into three states (stable, critical, and active) and the RG transformation acts on the energy transfer probabilities $p_{i}[11]$. The scheme is then extended in order to treat explicitly the four-state probability densities $n_{\alpha}$, which can be obtained self-consistently. The fixed point values of $n_{\alpha}$ and $p_{i}$ are in good agreement with exact results. In addition, we compute the critical exponent $\tau$ describing the avalanche size distribution (Table IV). The result is in good agreement with numerical and analytical estimations and appears to be robust with respect to the different approximations. Finally, we study the boundary scaling of the sandpile model, obtaining results in good agreement with exact results.

\section{ACKNOWLEDGMENTS}

E.V.I. and A.M.P. are grateful to V.B. Priezzhev for fruitful discussions. A.V. is indebted to J.M.J. van Leeuwen for very interesting discussions. A.V. and S.Z. thank V. Loreto and L. Pietronero, with whom they have collaborated on part of the work described here. A.M.P. and E.V.I. were partially supported by the Russian Foundation for Basic Research under Grant No. 97-01-01030. A.M.P. gratefully acknowledges support from the INTAS Grant No. 96-0457 within the research program of the International Center for Fundamental Physics in Moscow, and INTAS Grant No. N96-690. A.V. and S.Z. acknowledge partial support from the European Network under Contract No. ERBFMRXCT980183. 
[1] Phase Transition and Critical Phenomena, edited by C. Domb and M. S. Green (Academic Press, London, 1972-1976), Vols. 1-6; Phase Transition and Critical Phenomena, edited by C. Domb and J. L. Lebowitz (Academic Press, London, 19831995), Vols. 7-17.

[2] S. Katz, J.L. Lebowitz, and H. Spohn, Phys. Rev. B 28, 1655 (1983); J. Stat. Phys. 34, 497 (1984).

[3] B. Schmittmann and R. K. Zia, in Phase Transition and Critical Phenomena (Ref. [1]), Vol. 17.

[4] T. Vicsek, Fractal Growth Phenomena (World Scientific, Singapore, 1992).

[5] B. B. Mandelbrot, The Fractal Geometry of Nature (Freeman and Company, New York, 1983).

[6] P. Bak, C. Tang, and K. Wiesenfeld, Phys. Rev. Lett. 59, 381 (1987); Phys. Rev. A 38, 364 (1988).

[7] For a review, see, e.g., P. Bak and M. Creutz, in Fractals and Disordered Systems, edited by A. Bunde and S. Havlin (Springer-Verlag, Heidelberg, 1993), Vol. II; G. Grinstein, in Scale Invariance, Interfaces and Non-Equilibrium Dynamics, edited by A. McKane et al., Vol. 344 of NATO Advanced Study Institute, Series B: Physics (Plenum, New York, 1995).

[8] P. Bak, K. Chen, and C. Tang, Phys. Lett. A 147, 297 (1990).

[9] B. Drossel and F. Schwabl, Phys. Rev. Lett. 69, 1629 (1992).

[10] A. Vespignani and S. Zapperi, Phys. Rev. Lett. 78, 4793 (1997); Phys. Rev. E 57, 6345 (1998); R. Dickman, A. Vespignani, and S. Zapperi, ibid. 57, 5095 (1998).

[11] L. Pietronero, A. Vespignani, and S. Zapperi, Phys. Rev. Lett. 72, 1690 (1994); A. Vespignani, S. Zapperi, and L. Pietronero, Phys. Rev. E 51, 1711 (1995).

[12] V. Loreto, L. Pietronero, A. Vespignani, and S. Zapperi, Phys. Rev. Lett. 75, 465 (1995).

[13] A. Vespignani, S. Zapperi, and V. Loreto, Phys. Rev. Lett. 77, 4560 (1996); J. Stat. Phys. 88, 47 (1997).
[14] E.V. Ivashkevich, Phys. Rev. Lett. 76, 3368 (1996).

[15] J. Hasty and K. Wiesenfeld, J. Stat. Phys. 86, 1179 (1997).

[16] A. Ben-Hur, R. Hallgass, and V. Loreto, Phys. Rev. E 54, 1426 (1996).

[17] J. Hasty and K. Wiesenfeld, Phys. Rev. Lett. 81, 1722 (1998).

[18] T. Tomé and M.J. de Oliveira, Phys. Rev. E 55, 4000 (1997); M.J. de Oliveira and J.E. Satulovsky, ibid. 55, 6377 (1997).

[19] Y.C. Zhang, Phys. Rev. Lett. 63, 470 (1989); L. Pietronero, P. Tartaglia, and Y. C. Zhang, Physica A 173, 129 (1991).

[20] S.S. Manna, J. Phys. A 24, L363 (1991).

[21] P. Grassberger and S.S. Manna, J. Phys. (France) 51, 1077 (1990).

[22] A.L. Stella, C. Tebaldi, and G. Caldarelli, Phys. Rev. E 52, 72 (1995).

[23] S. Lübeck and K.D. Usadel, Phys. Rev. E 55, 4095 (1997); S. Lübeck, ibid. 56, 1590 (1997).

[24] D. Dhar, Phys. Rev. Lett. 64, 1613 (1990).

[25] S.N. Majumdar and D. Dhar, J. Phys. A 24, L357 (1991).

[26] V.B. Priezzhev, J. Stat. Phys. 74, 955 (1994).

[27] E.V. Ivashkevich, J. Phys. A 27, 3643 (1994).

[28] E.V. Ivashkevich, D.V. Ktitarev, and V.B. Priezzhev, J. Phys. A 27, L585 (1994).

[29] V.B. Priezzhev, D.V. Ktitarev, and E.V. Ivashkevich, Physica A 209, 347 (1994).

[30] V.B. Priezzhev, D.V. Ktitarev, and E.V. Ivashkevich, Phys. Rev. Lett. 76, 2093 (1996).

[31] T. Niemeijer and J.M.J. van Leeuwen, in Phase Transition and Critical Phenomena (Ref. [1]), Vol. 6.

[32] R. Dickman, Phys. Rev. A 38, 2588 (1988).

[33] A. Ben-Hur and O. Biham, Phys. Rev. E 53, R1317 (1996); E. Milshtein, O. Biham, and S. Solomon, ibid. 58, 303 (1998).

[34] J.L. Cardy, in Phase Transition and Critical Phenomena (Ref. [1]), Vol. 11. 